\title{
Amebas testáceas: ocorrência e distribuição Baía de Iguape/BTS - Bahia, Brasil
}

\author{
Cláudia Ferreira da Cruz \\ Faculdade Área 1, De Vry Brasil \\ Avenida Luiz Viana, 3172, Paralela, CEP 41720-200, CEP 2106-3911, Salvador - BA, Brasil \\ cfcruz@gmail.com
}

Submetido em 12/10/2011

Aceito para publicação em 12/05/2012

\section{Resumo}

A Baía de Iguape $\left(12^{\circ} 00^{\prime}-13^{\circ} 00^{\prime} \mathrm{S}\right.$ e $\left.38^{\circ} 30^{\prime}-39^{\circ} 30^{\prime}\right)$ é uma região estuarina está localizada na foz do rio Paraguaçu, no interior da Baía de Todos os Santos (BTS), Bahia, Brasil. Situada a 30km a jusante da Barragem Pedra do Cavalo, a segunda maior barragem no Brasil. A qualidade das águas está sob forte influência da maré oceânica, originada da BTS e das descargas do rio Paraguaçu. Esta área foi escolhida para o presente estudo, devido a sua complexidade ambiental. Nela, os estresses naturais típicos das zonas parálicas encontram-se intensificados pela presença de vários fatores abióticos, destacando-se as correntes oceânicas e as variações de vazão da descarga fluvial estabelecidas, principalmente, pela represa Pedra do Cavalo. Foram determinadas as densidades de amebas testáceas vivas e mortas, por volume de sedimento, identificando as espécies, calculados o índice de diversidade, a constância, a equitatividade, a riqueza, as quantificações e qualificações dos estados de preservações das testas nos perfis e análise sedimentológica.

Palavras-chaves: Amebas testáceas; Barragem; Maré; Região estuarina; Sedimentos

\section{Abstract}

Thecamoebians: occurrence and distribution in Iguape Bay/BTS - Bahia, Brazil. The Iguape Bay $\left(12^{\circ} 00^{\prime}-13^{\circ} 00^{\prime} \mathrm{S}\right.$ and $\left.38^{\circ} 30^{\prime}-39^{\circ} 30^{\prime}\right)$ is an estuary located at the mouth of the Paraguaçu River, inside Todos os Santos Bay (BTS), in Bahia, Brazil. It is $30 \mathrm{~km}$ downstream from the Pedra do Cavalo Dam, which is the second largest dam in Brazil. The water in Iguape Bay is influenced by ocean tides originating from the BTS and the discharge of the Paraguaçu River. The effects of the currents as well as the variation in the outflow from the Pedra do Cavalo Dam have intensified the natural stress of this paralic environment. The main goal of this study was to investigate the distribution of Thecamoeba species present in the bottom sediments of Iguape Bay, in order to produce data that can be used to interpret the processes operating in this ecologically complex environment. The density of the living and dead species of Thecamoeba was determined by the volume of sediment collected, and the diversity index, constancy, evenness and richness of Thecamoeba were calculated. Sedimentological analyses were also performed in order to classify sediment types in the selected profiles.

Key words: Dam; Estuary; Sediments; Thecamoebians; Tides 


\section{Introdução}

Os estuários são enriquecidos e influenciados pelos domínios continental e oceânico. Estes ambientes abrigam ecossistemas muito ricos e diversificados, constituindo-se, assim, em zonas privilegiadas para a reprodução e o desenvolvimento de numerosas espécies marinhas e dulciaquícolas, mantendo o equilíbrio ecológico das regiões costeiras (EICHLER-COELHO et al., 1997). Os estudos sobre a microfauna parálica de amebas testáceas em ambientes preservados são importantes, pois, além de fornecerem informações mais precisas sobre o funcionamento hidrodinâmico da região estudada, podem auxiliar a compreender as complexas relações existentes entre associações microfaunísticas e parâmetros abióticos (DULEBA et al., 1999).

Geologicamente estes organismos são importantes por possuírem testas que se incorporam ao sedimento quando os mesmos morrem ou se reproduzem (PHLEGER, 1960a; 1960b), além de apresentar grãos de sedimentos formando essas estruturas. Como parte integrante do sedimento, suas testas passam a se comportar como grãos sedimentares sendo transportadas ou erodidas, permitindo assim o registro de condições físicas e químicas além de eventos que ocorrem nos ambientes parálicos (ROCHA, 1972; MURRAY, 1991; DULEBA, 1994; BONETTI, 1995; 2000).

O grupo dos arcelinideos foi estudado ao longo do último século, contudo os pesquisadores só reconheceram sua utilidade como indicadores ambientais e paleo-ambientais nos últimos dez anos (ARNDT, 1993; KUMAR; DALBY, 1998; PATTERSON; KUMAR, 2002). Foi possível, ao longo desse período, estabelecer que várias espécies de amebas testáceas habitam, preferencialmente, ambientes específicos que apresentem fatores abióticos ideais para o seu desenvolvimento, devido ao fato de serem sensíveis a variações de fatores físico e químicos (CLOSS, 1962; CLOSS; MADEIRA, 1967; GREEN, 1975; WALTER, 1982; BONETTI, 1995; ESCOBAR; MARTINEZ, 2002). Esses protozoários são abundantes na maioria dos ecossistemas aquáticos continentais, possuem uma alta variação de características que os tornam ferramentas importantes nas abordagens ecológicas, apresentam grande produção de biomassa. O seu tamanho e tempo de geração permitem investigações sobre processos demográficos, tanto em escala espacial, quanto temporal ocorrem tanto em ambientes de outros países (SCHÖNBORN, 1973; COLLINS et al., 1990; HARDOIM; HECKMAN, 1996; HARDOIM, 1997), bem como em ambientes fluviais e estuarinos do território nacional (ZUCON; LOYOLLA E SILVA, 1992; VELHO; LANSAC-TÔHA, 1996; LANSACTOHA et al., 2004; 2007; VELHO et al., 1996; 1999; 2003). Devido à importância ecológica destes organismos, este artigo tem como objetivo registrar a ocorrência e distribuição das amebas testáceas na Baía de Iguape/BTS/Bahia.

\section{Material e Métodos}

Durante o mês de novembro de 2002 foram coletadas 176 amostras de sedimento de superfície de fundo, abrangendo o setor norte até o setor sul da Baía de Iguape. Do total dessas amostras, apenas 76 foram selecionadas para realização deste trabalho, uma vez que, Carvalho (2000) dividiu o estuário tectônico em setores diferenciados que pode ser citado como: norte, centro e sul. As 76 amostras coletadas, para o estudo em questão, foram distribuídas em 11 perfis, localizados na região central devido à influência dos dois fluxos distintos de águas (e.g. doce e salgada) e apresentam a orientação no sentido Oeste para Leste como pode ser observado na Figura 1. Essa distribuição, para análise dos resultados deste trabalho, foi adotada devido ao fato do sentido oposto apresentados pela descarga fluvial determinada pela barragem Pedra do cavalo e da maré oceânica oriunda da BTS.

As amostras foram coletadas pontualmente, em duplicata, utilizando-se o Van Veen, e acondicionadas em frascos plásticos contendo solução de álcool a $80 \%$ e corante rosa de Bengala (1g/L de álcool) (WALTON, 1952). A função do álcool é impedir o ataque bacteriano (EICHLER et al., 2001). Posteriormente, foram lavadas sob água corrente, para retirar o excesso do corante, em peneira com espaçamento de malha de $0,062 \mathrm{~mm}$. De cada amostra foi feita uma subamostragem com $10 \mathrm{~g}$ de sedimento, após a secagem do sedimento, ao ar livre. 
Das sub-amostras, foram realizadas as quantificações, as qualificações (vivos, com protoplasmas corados; morto, sem coloração) e a identificação, em nível de espécie, de todas as amebas testáceas presentes nessa quantidade de sedimento (SEMENSATTO-JR, 2003). A análise do total desse protozoário é baseada na afirmação de Scott et al. $(1980 ; 1990)$ de que os dados da fauna total descrevem com maior precisão os ambientes atuais, e deste modo, são úteis para a maioria dos estudos ambientais tanto ecológicos, bem como, os geológicos. Ainda dessas amostras foram feitas as análises granulométricas. As identificações das espécies foram feitas por Loeblich e Tappan (1968; 1978) e Medioli e Scott (1988).

FIGURA 1: Localização dos pontos amostrados em 11 perfis e as amostras que os compõem.

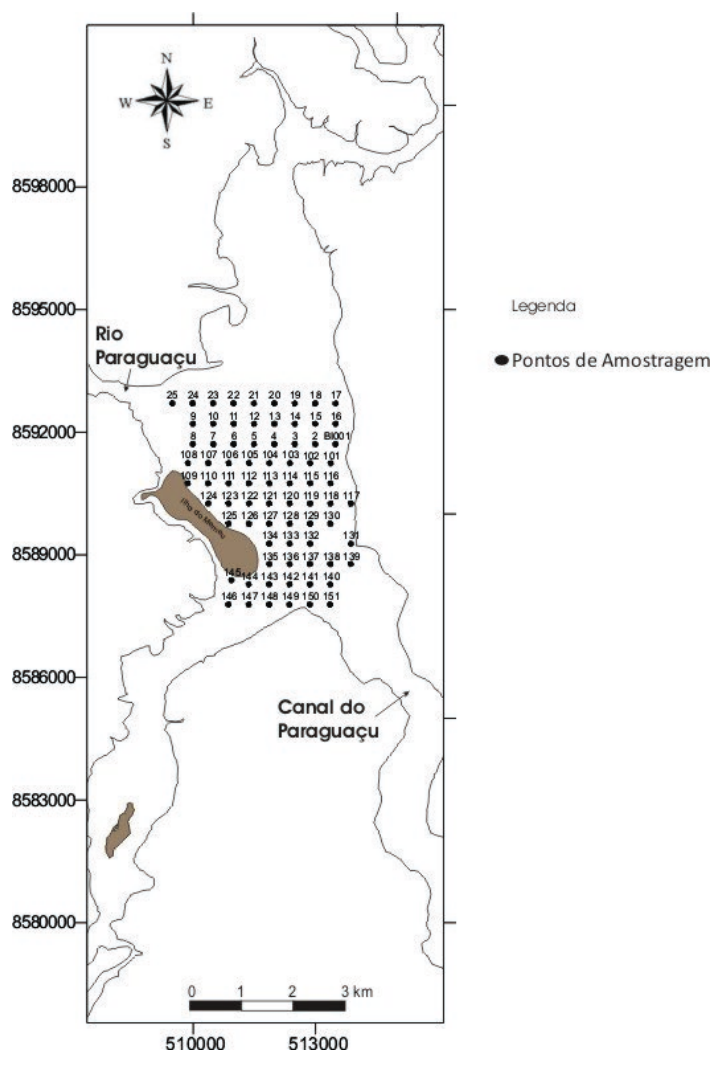

A partir dos dados obtidos com a análise da fauna de amebas testáceas (números de indivíduos por espécies) foram calculados com o programa Statistic 5.5 (módulo Basic Statistic), bem como seguindo as metodologias propostas por Shannon (1948), Margalef (1958), Dajoz (1983), Tinoco (1989), Clarke e Warwick (1994), Valentin (2000) e Semensatto-Jr (2003), foram realizadas as seguintes análises: frequência relativa (\%), frequência de ocorrência (\%), índices de diversidade (H'), riqueza (R) e equitatividade ( $\left.\mathrm{J}^{\prime}\right)$.

\section{Área de estudo}

A Baía de Iguape pode ser classificada como um estuário tectônico, que pode ser definido inicialmente em costas tectonicamente ativa, tem origem associada a processos tectônicos, com falhamentos, vulcanismos e movimentos isostáticos (CARVALHO, 2000). Este tipo de estuário apresenta consideráveis variações morfológicas, mas os processos oceanográficos podem ser semelhantes aos atuantes em outros tipos de estuários (KJERFVE, 1989).

A descarga fluvial proveniente do Rio Paraguaçu sofre variação no volume de água conforme vazão proposta pela Embasa que coordena o funcionamento da barragem Pedra do Cavalo (MESTRINHO, 1998) determinando desta forma as velocidades de correntes diferenciadas na região. A maré iniciada no oceano Atlântico passando pela BTS (valor da altura média $2,25 \mathrm{~m}$ ), que faz sentir-se nas proximidades da cidade de Cachoeira (valor da altura média $2,68 \mathrm{~m}$ ), pois percorre todo o Canal do Paraguaçu (antigo Canal de São Roque, conduto para a maré oceânica (CARVALHO, 2000)), a região central da Baía de Iguape e parte da calha do Rio Paraguaçu (LESSA et al., 2001). O valor dessa altura média permite classificar este estuário lagunar como de mesomaré em consonância a Davies (1964).

\section{Resultados e Discussão}

A fauna bentônica de amebas testáceas presente em 11 perfis foi composta por 15 espécies, compreendendo 10 gêneros, três superfamílias (Figura 2). A superfamília Arcellacea, além de apresentar um maior número de representantes, foi também a subordem mais representativa em número de indivíduos (749), seguidas pelas superfamílias Euglyphacea (14) e Cryptodifflugiacea (um) (MEDIOLI et al., 1990).

$\mathrm{Na}$ região central do estuário tectônico ocorre a predominância de sedimentos arenosos como pode ser observado na Figura 3. 
Dentre os 764 espécimes identificados, destacamse como espécies dominantes em cada perfil as listadas abaixo com os valores específicos das frequências relativas de cada uma delas, estes valores estão registrados nos parênteses após os respectivos nomes: no perfil 01 Centropyxis aculeata - Ehrenberg, $1835(30,8)$, Difflugia protaeiformis - Lamarck, $1816(23,1)$ e Nebala longicollis - Penard, 1890 (46,2); no perfil 02 Difflugia protaeiformis - Lamarck, 1816 (50) e Phryganella nidulus - Penard, 1902 (50); no perfil 03 não existe amebas testáceas; no perfil 04 Difflugia protaeiformis Lamarck, 1816 (73,3) e Diplochlamys leidyi - Greeff, 1888 (10,5); no perfil 05 Cochliopodium pellucidum Hertwig \& Lesser, 1874 (27,6), Difflugia protaeiformis Lamarck, 1816 (14,3), Euglypha tuberculata - Dujardin, 1841 (10,5), Gocevia pontica - Valkanov, $1972(13,3)$ e Penardochlamy arcelloides - Penard, $1904(17,1)$; no perfil 06 Difflugia protaeiformis - Lamarck, $1816(56,3)$; no perfil 07 Chlamydamoeba tentaculifera-Collin, 1912 $(29,2)$, Difflugia protaeiformis - Lamarck, $1816(44,8)$ e Gocevia pontica - Valkanov, $1972(20,1)$; no perfil 08 Difflugia protaeiformis - Lamarck, $1816(65,9)$; no perfil 09 Chlamydamoeba tentaculifera - Collin, 1912 $(23,1)$; Difflugia protaeiformis - Lamarck, $1816(30,8)$ e Gocevia pontica - Valkanov, $1972(38,5)$; no perfil 10 Difflugia protaeiformis - Lamarck, $1816(89,5)$ e Gocevia pontica - Valkanov, 1972 (10,5); no perfil 11 Difflugia protaeiformis - Lamarck, $1816(93,8)$ (Figura 4). Ainda na Figura 4, as espécies consideradas "Outras" foram as abundantes, frequentes, pouco frequentes e raras dos perfis, que por sua vez, apresentaram variações em quantidade diferenciadas para cada um deles.

FIGURA 2: Número de indivíduos, em porcentagem, das superfamílias de amebas testáceas.

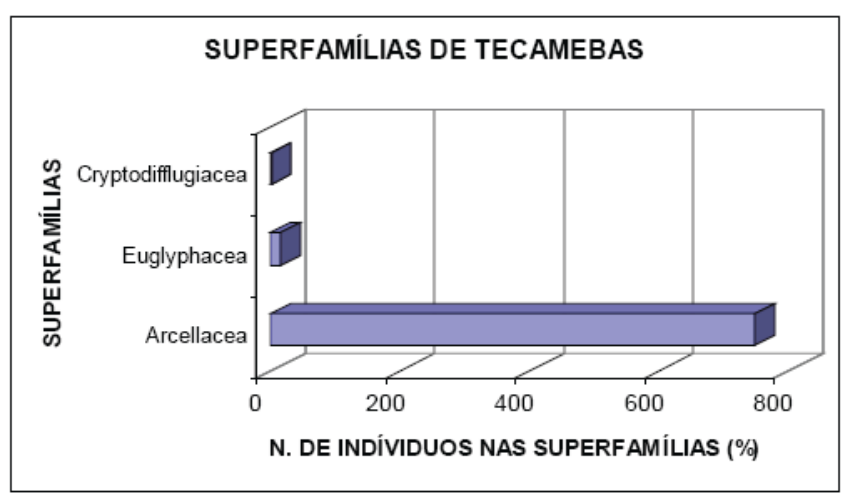

Revista Biotemas, 25 (3), setembro de 2012
FIGURA 3: Porcentagem de sedimentos na região central da Baía de Iguape/BTS .

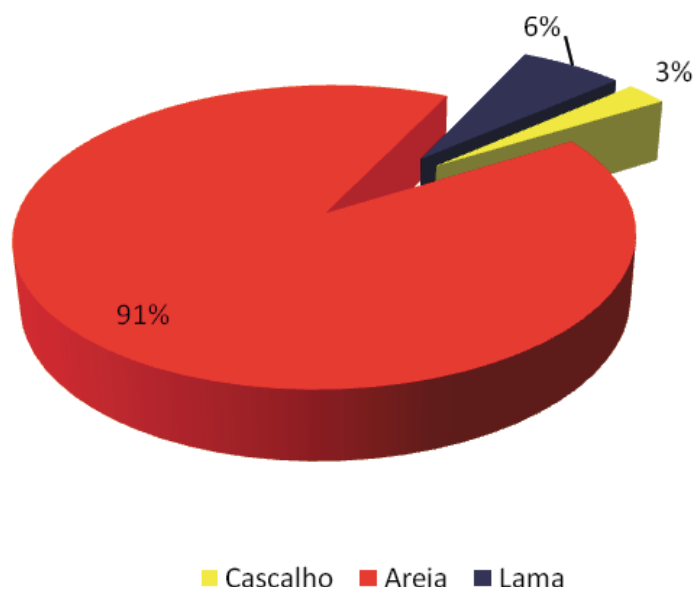

Analisando a abundância relativa das espécies por perfil foi possível estabelecer os indivíduos dominantes em cada perfil, bem como, perceber a diferença entre os perfis com relação aos percentuais variáveis em relação às espécies dominantes, bem como a não existência de espécies amebas testáceas no perfil 03, e o máximo de nove espécies no perfil 06. Contudo, ocorre uma variação de quantidade de espécies e tipos de espécies para cada perfil. No perfil 05 foram encontradas cinco espécies distintas, sendo que, neste perfil foram identificados os espécimes Cochliopodium pellucidum se reproduzindo como representado na Figura 5. Segundo Medioli e Scott (1988), as amebas testáceas se reproduzem assexuada e sexuadamente, isto dependo da espécie. O registro desse evento permite inferir que o microambiente, para estes espécimes, estava adequado para sobrevivência e adaptações.

Segundo Closs (1962), as amostras coletadas nas zonas com correntes fortes (ambientes lóticos) mostram pobres, enquanto que as amostras coletadas em zonas protegidas pela topografia dos arredores (ambientes lênticos) apresentam ricas em protozoofauna. Em contra partida, BONECKER et al. (2000) relatam que as amebas testáceas apresentam um padrão nítido de variação espacial de riqueza devido às diferenças do fluxo da corrente fluvial, ou seja, maiores em ambientes lóticos, e menores em ambientes lênticos. O mesmo pode ser observado por LANSAC-TÔHA et al. (1997) relatam que devido à velocidade do fluxo fluvial interfere na presença 
FIGURA 4: Espécies de amebas testáceas dominantes, em cada perfil, na área de estudo.

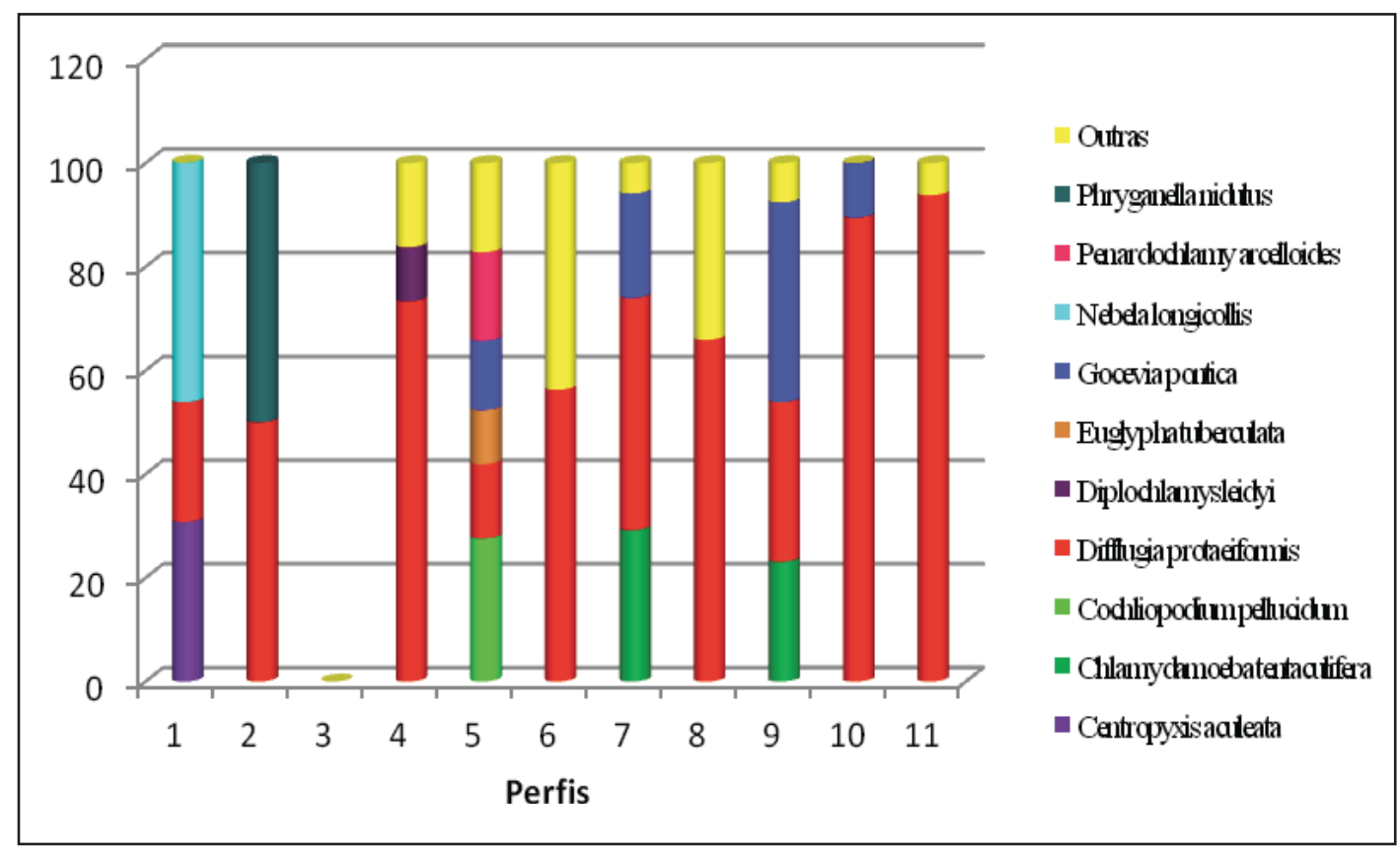

desses organismos, haja vista que em ambientes lóticos, com maior velocidade de água ocorre a ressuspenção da matéria orgânica para a coluna de água, visto que o grupo é associado ao sedimento, propiciando assim o bom desenvolvimento para as amebas testáceas devido à disponibilidade de alimento.

Comparando-se os índices analisados nos perfis, os valores máximos e mínimos foram obtidos, respectivamente, em relação à diversidade de 1,83 no perfil 05 e 0,19 no perfil 11. Em relação à riqueza de 1,80 no perfil 06 e 0,34 no perfil 10 ; em relação à equitatividade de 0,94 no perfil 05 e 0,27 no perfil 11 . Foi observado que o índice de riqueza apresentou-se elevado em relação ao valor mínimo. Correlacionado isto ao índice de diversidade reflete as características adversas encontradas em alguns microambientes formados na área para a microfauna analisada. Os dados obtidos sobre a quantificação total das amebas testáceas, em cada perfil, mostram que existem microambientes propício para o desenvolvimento desse protozoário. Em relação às amebas testáceas, a representatividade significante no ambiente estudado se localiza nos perfis 04, 05, 06 e 07 parte central do estuário tectônico, com características estuarinas.
As distribuições dos índices de diversidade, riqueza e equitatividade, em cada perfil, estão apresentadas na Figura 6. Em relação à fauna de amebas testáceas os índices de diversidades foram maiores nos perfis 05 , 06,07 . Contudo o índice de riqueza foi maior nos perfis $02,05,06$ e 08 .

A diversidade específica encontrada pode ser considerada baixa (10 gêneros e 15 espécies de amebas testáceas). Este pode ser um indício das condições ecológicas mais restritivas existentes neste ambiente. Comparando-se estes valores com os outros autores de ambientes costeiros, encontramos valores aproximados dos valores assinalados em zonas estuarinas do Rio Itapitangui (BONETTI; EICHLER, 1997) e ao Estuário de Guaratuba (BARBOSA, 1995), ambos com maior influência fluvial e com valores de diversidade inferiores a 30 espécies. No Rio Pojuca (ANDRADE et al., 1999) apresentou uma diversidade baixa 17 espécies.

A análise da frequência de ocorrência de amebas testáceas, em cada perfil, mostra uma variação entre os três índices analisados. Nos perfis 01,02 e 11 só ocorrem espécies acidentais, enquanto nos outros perfis apresentaram espécies constantes, acessórias e acidentais em percentuais diferenciados. Com exceção 
do perfil 03 que não apresentou nenhuma espécie, sendo considerado inóspito para o desenvolvimento dessas espécies (Figura 7). Como os perfis anteriormente citados estão localizados nas extremidades das áreas analisadas, as amebas testáceas ali encontradas podem ser resultado do transporte pelas correntes oceânicas e fluviais existentes na região.

FIGURA 5: Prancha com as fotos das espécies que são elas: Foto 7: Difflugia globularis Wallich, 1864, fixada em fragmento rochoso; Foto 8 - Euglypha tuberculata Dujardin, 1841; Foto 9 - Cochliopodium pellucidum Hertwig \& Lesser, 1874; Foto 9b - Cochliopodium pellucidum Hertwig \& Lesser, 1874. Espécie em foco, aderida em fragmento de rocha (organismo séssil); Foto 9c - Cochliopodium pellucidum Hertwig \& Lesser, 1874. Membrana de aderência do organismo, ligada à testa membranácea com grãos aderidos. Espécie se apresentava corada. (organismo descolado do fragmento com bisturi); e, Foto 9d-Cochliopodium pellucidum Hertwig \& Lesser, 1874. No interior do organismo, embaixo da membrana de aderência foram encontradas 8 testas com mesmo formato, todas coradas. Essas testas menores foram encontradas no segundo organismo descolado de outro grão de sedimento com bisturi (fotomicrografias disponíveis nas pranchas em CRUZ, 2004). As testas menores não apresentavam grãos aderidos e possuíam flexibilidade.

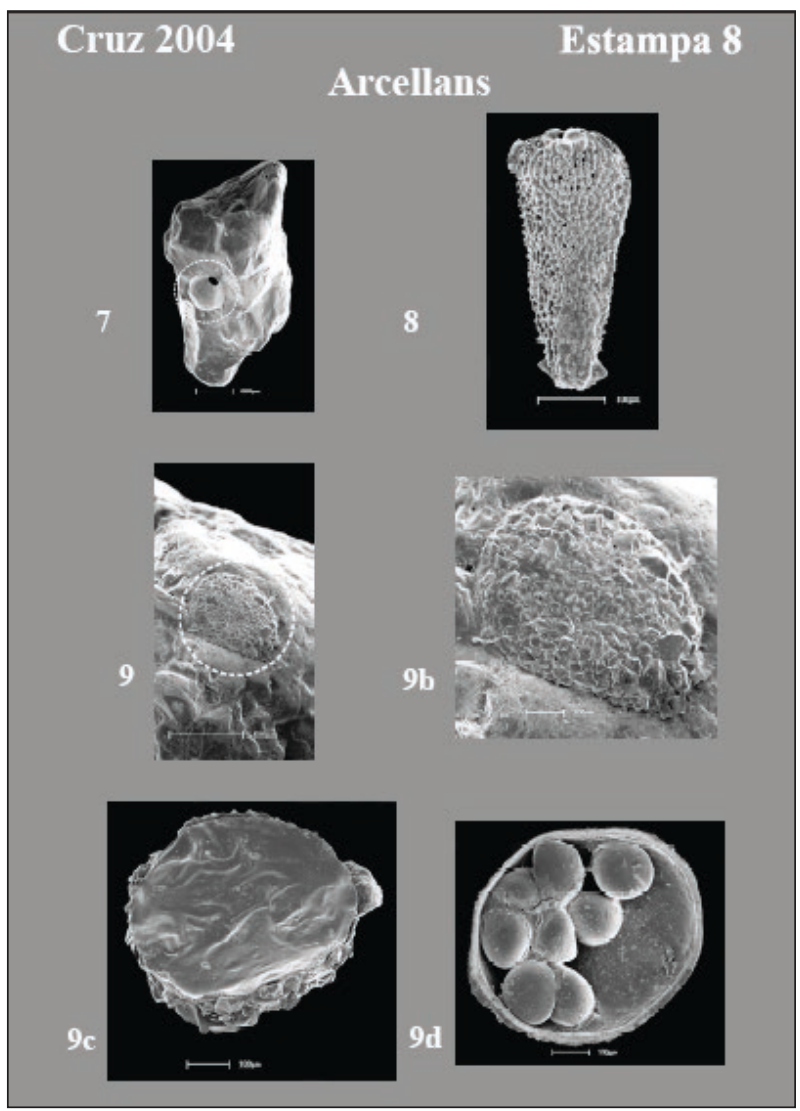

FIGURA 6: Representação gráfica da diversidade, riqueza e equitatividade nos perfis.

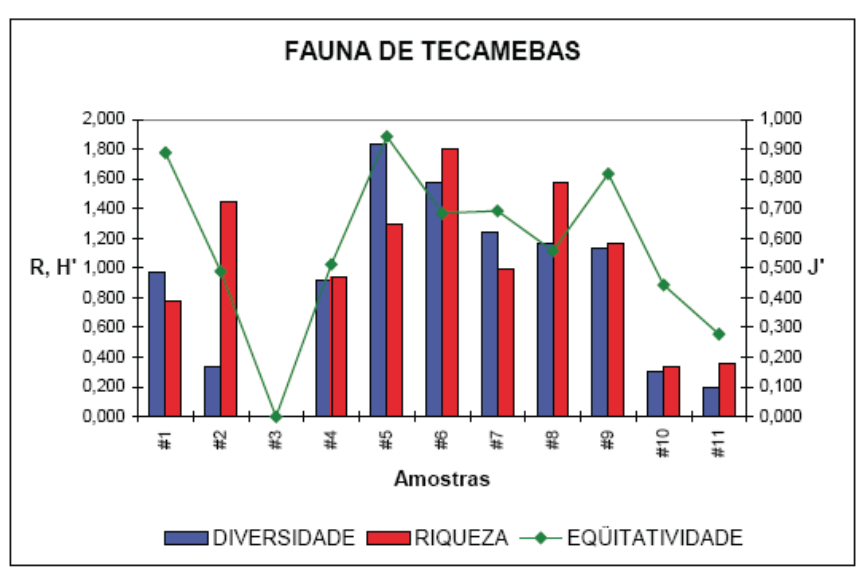

FIGURA 7: Representação gráfica da frequência de ocorrência em cada perfil.

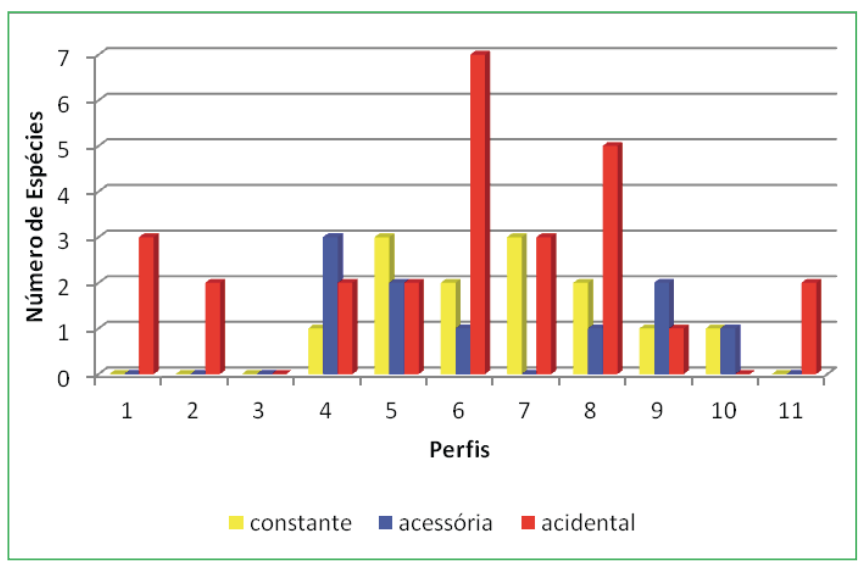

Os números de indivíduos vivos (protoplasma corados) e mortos são representados para cada perfil na Figura 8. Embora haja, de forma bem significante, representantes corados nos perfis 04, 05 e 07, nestes mesmos perfis há concomitantemente, representatividade em testas não coradas. As testas não coradas foram consideradas organismos mortos. Das espécies coradas, consideradas vivas neste estudo, podemos citar Diplochlamys leidyi, Difflugia protaeiformis, Cochliopodium pellucidum e Gocevia pontica. Levando em consideração que seis espécimes de Cochliopodium pellucidum foram encontrados se reproduzindo, com todos os indivíduos corados nos perfis anteriormente citados foi possível inferir a boa adaptação da espécie na área. 
FIGURA 8: Representação gráfica das amebas testáceas coradas e não corados nos perfis.

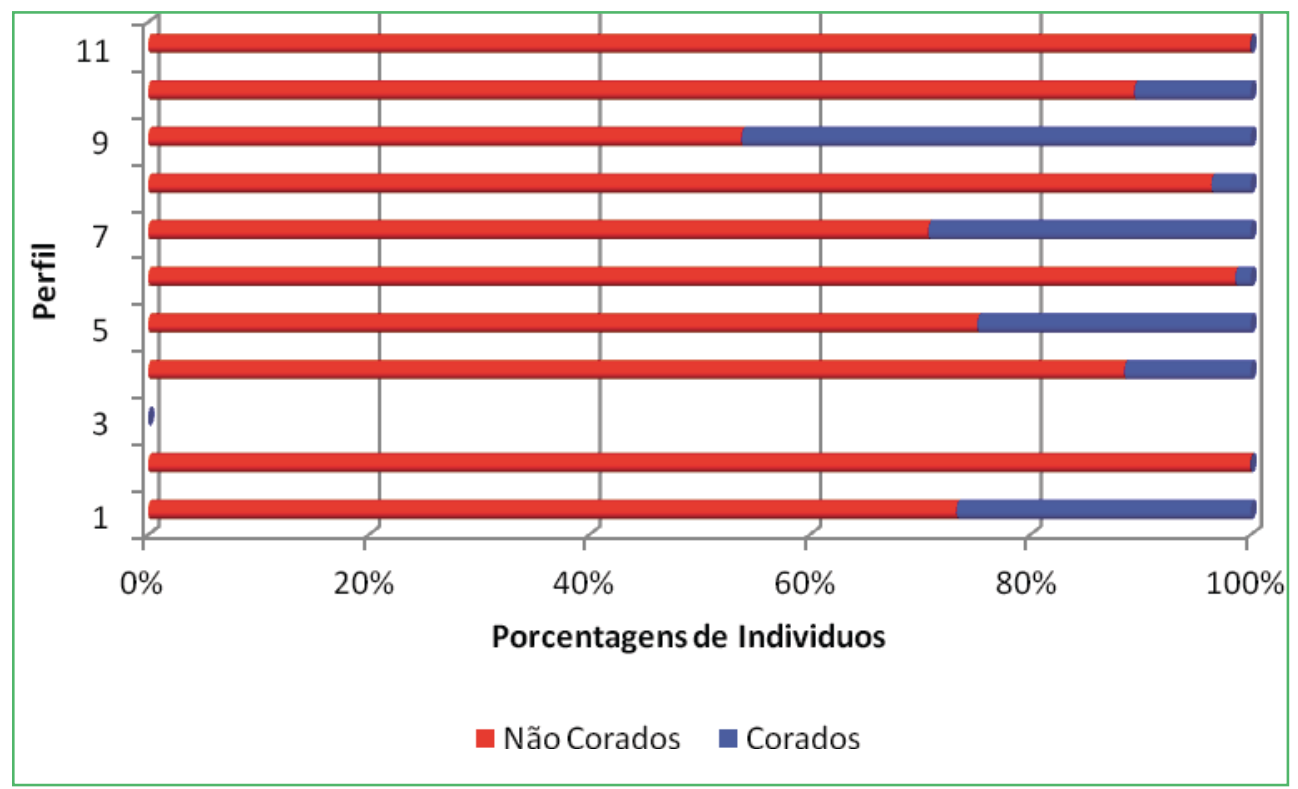

O efeito da descarga fluvial, constantemente adicionada pelo rio, além de gerar um componente da circulação estuarina que naturalmente se desloca estuário abaixo, ao diluir a água do mar produz diferenças de densidade ao longo do estuário, gerando movimentos estuário acima forçados pelo gradiente de pressão. A interação das várias propriedades e processos - descarga (ou vazão) do rio, correntes de maré, gradiente de pressão, advecção difusão turbulenta - produz, dentro da delimitação geomorfológica da bacia estuarina, a distribuição de salinidade que é características de cada estuário (OFFICER, 1983, apud MIRANDA et al., 2002).

As amebas testáceas pertencentes ao meiobentos têm sua distribuição espacial afetada diretamente pelas características abióticas dos sedimentos e da coluna d’água adjacentes. Variáveis como a salinidade, o $\mathrm{pH}$, a granulometria e os teores orgânicos dos sedimentos têm demonstrado desempenhar importante papel na estruturação das populações bentônicas, superando muitas vezes condicionantes biológicas como a predação e a simbiose (BOLTOVSKOY, 1964; BOLTOVSKOY; WRIGHT, 1976; MURRAY, 1991; DEBENAY et al., 1996a; 1996b). Alguns estudos também têm buscado compreender a interferência dos poluentes na estrutura das comunidades biológicas e na fisiologia de algumas espécies (SHARIFI et al., 1991; YANKO et al., 1994; ALVE, 1995).
Os números de amebas testáceas membranáceas, aglutinantes e silicosas são representados para cada perfil na Figura 9. Existe, nos perfis 01, 02 e 08, uma baixa representatividade dos organismos com testas aglutinantes. Os indivíduos silicosos estão representados nos perfis 01, 04, 05, 06 e 08, estes possivelmente devem usar a sílica disponível no meio para confecção de suas testas, e, dos membranáceos, com testas de pseudoquitina, presentes nos perfis $01,04,05,06,07$, 08 e 09 , que usam o substrato como base para formação e fixação de suas testas.

FIGURA 9: Representação gráfica dos tipos de testas das amebas testáceas nos perfis.

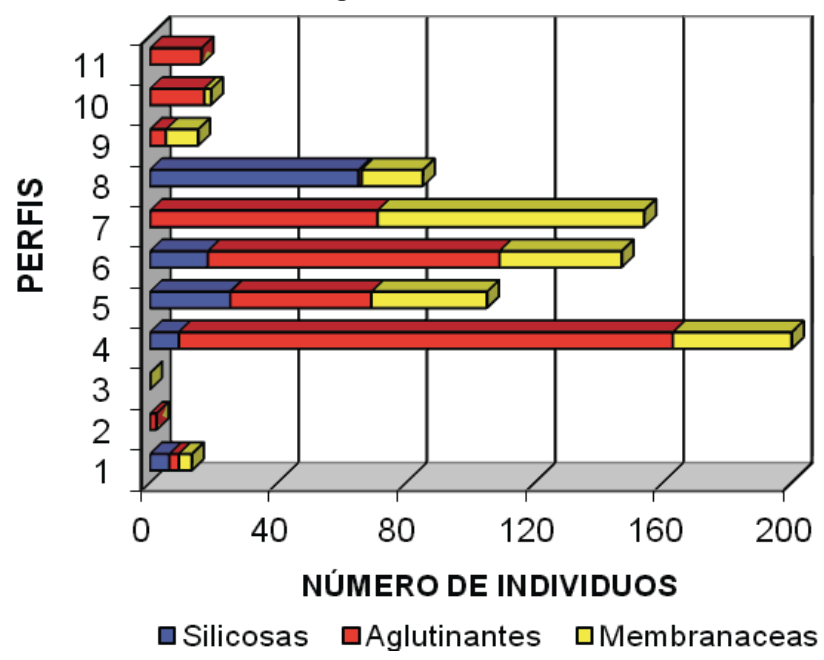


As amebas testáceas estavam presentes, em abundância, nos perfis centrais que são: 04, 05, 06, 07 e 08. Devido à representatividade desses organismos, nestes perfis, foi possível sugerir a presença do aporte de água doce constante nesta região, tendo desta forma um canal preferencial de circulação dessa água doce no ambiente.

Masselink et al. (1999) e Anjos et al. (2001) inferem que as testas, em bom estado de conservação e retrabalhada, sugerem que os sedimentos são transportados para a área do estuário a curta distância. Ainda segundo os primeiros autores, as concentrações altas de fragmentos de testas aglutinantes em determinada área indicam fortes correntes. Fator confirmado pelos valores de correntes apresentados por Genz e Lessa (2002) na área.

As espécies das amebas testáceas dominantes na área estudada foram: Centropyxis aculeata, Chlamydamoeba tentaculifera, Cochliopodium pellucidum, Difflugia protaeiformis, Diplochlamys leidyi, Euglypha tuberculata, Gocevia pontica, Nebela longicolis, Penardochlamy arcelloides e Phryganella nidulus.

O presente estudo permitiu uma caracterização geral da ocorrência e distribuição das amebas testáceas, na área da Baía de Iguape e Baixo Curso do Rio Paraguaçu (região central), e isso possibilita a seleção de determinados locais para monitoramento ou controle da população desses organismos, além disso, fornece subsídios para pesquisa em outros ambientes.

A presença de amebas testáceas se reproduzindo permitem inferir a existências de microambientes favoráveis ao seu desenvolvimento.

\section{Agradecimentos}

Ao CNPq, pela concessão da bolsa de Mestrado; ao Prof. Guilherme Camargo Lessa, do IGEO - UFBA, por ter cedido às amostras para realização desse trabalho de pesquisa; à Prof ${ }^{a}$ Zelinda Margarida de Andrade Nery Leão, do IGEO - UFBA, por ter financiado as microfotografias em MEV.

\section{Referências}

ALVE, E. Benthic foraminiferal responses to estuarine pollution: a review. Journal Paleontology, Tulsa, v. 25, n. 3, p. 190-204, 1995.

ANDRADE, A. J.; ANJOS, G. S.; KIKUCHI, R. K. P. Ocorrência e variação sazonal da fauna de foraminíferos do Rio Pojuca, Bahia. In: ENCOTRO DE ZOOLOGIA DO NORDESTE, XII, 1999, Salvador. Resumos... Salvador: ENCOTRO DE ZOOLOGIA DO NORDESTE, 1999. p. 65-67.

ANJOS, G. S.; LESSA, G. C.; ANDRADE, E. J.; MACHADO, A. J. Utilização de foraminíferos bentônicos como indicadores de transporte de sedimentos: o caso do Estuário de Cacha - Prego, Bahia. In: CONGRESSO DA ASSOCIAÇÃO BRASILEIRA DE ESTUDOS DO QUATERNÁRIO, VIII, 2001, Imbé. Resumos... Imbé: ABEQUA, 2001. p. 57-59.

ARNDT, H. A critical review of the importance of Rhizopods (Nakes and Testate Amoebae) and Actinopods (Heliozoa) in Lake Plankton. Marine Microbiology Food Webs, Paris, v. 7, p. 3-29, 1993.

BARBOSA, C. F. Foraminífera e Arcellacea ("Thecamoebia") recentes do Estuário de Guaratuba, Paraná, Brasil. Anais da Academia Brasileira de Ciências, Rio de Janeiro, v. 67, n. 4, p. 465-492, 1995.

BOLTOVSKOY, E. Seasonal occurrences of some living foraminifera in Puerto Deseado (Patagonica, Argentina). Journal du Conseil/Conseil Permanent International pour l'Exploration de la Mer, Paris, v. 29, n. 2, p. 136-145, 1964.

BOLTOVSKOY, E.; WRIGHT, R. Recent Foraminifera. The Hague: Junk, 1976. 515 p.

BONECKER, C. C.; VELHO, F. M.; COSTA, C. L.; PEREIRA, D. G.; ALVES, G. M.; MACHADO, R. A. M.; LANSACTÔHA, F. A. Zooplâncton. 2000. Maringá: Nupélia. Relatório Técnico. Disponível em <www. Peld.uem.Br/Relat2000/2 2 CompBioticoZooplancton.PDF>. Acesso: 25 maio 2002.

BONETTI, C. Associações de foraminíferos e amebas testáceas indicadoras de sub-ambiente na zona estuarina do Rio Itapitangui-Cananéia, SP. 1995. 150 f. Dissertação (Mestrado em Oceanografia) - Universidade de São Paulo, Instituto Oceanográfico, São Paulo. 1995.

BONETTI, C.; EICHLER, B. B. Benthic Foraminiferal and Thecamoebians as indicators of river/sea gradients in the estuarine zone of Itapitangui River - Cananéia, Brazil. Anais da Academia Brasileira de Ciências, Rio de Janeiro, v. 69, n. 4, p. 545-593, 1997.

BONETTI, C. V. D. H. C. Foraminíferos como bioindicadores do gradiente de estresse ecológico em ambientes costeiros poluídos. Estudo aplicado ao sistema estuarino de Santos - São Vicente (SP - Brasil). SP. 2000. 117 f. Tese (Doutorado em Oceanografia Geológica) - Universidade de São Paulo, Instituto Oceanográfico, São Paulo. 2000.

CARVALHO, J. B. Caracterização morfoestratigráficas do preenchimento sedimentar da Baía de Iguape, Bahia - Influência das variações eustáticas do nível do mar e atividades tectônicas recentes. 2000. 23 f. Dissertação (Mestrado em Geologia) Universidade Federal da Bahia, Salvador. 2000.

CLARKE, K. R.; WARWICK, R. M. Change in marine communities: an approach to statistical analysis and 
interpretation. Plymouth: Plymouth Marine Laboratory Report 1994. $144 \mathrm{p}$.

CLOSS, D. Foraminíferos e Amebas testáceas na Lagoa dos Patos (R.G.S.). Boletim N. 11. Porto Alegre: Gráfica da Universidade do Rio Grande do Sul. Escola de Geologia de Porto Alegre, 1962. $50 \mathrm{p}$.

CLOSS, D.; MADEIRA, M. Foraminíferos e Amebas testáceas aglutinantes da Lagoa de Tramandaí, no Rio Grande do Sul Iheringia, Zoologia, Porto Alegre, n. 35, p. 7-31, 1967.

COLLINS, E.; McCARTHY, F.; MEDIOLI, S.; SCOTT, B.; HONIG A. Biogeographic distribution of modern thecamoebians in a transect along the Eastern North American Coast. In: HEMLEBEN, C.; KAMINSKI, M. A.; KUHNT, W.; SCOTT, D. B. (Ed.). Paleotology, Biostratigrafy, Paleocology, Taxa Agglutinants Foraminifer. Netherlands: Klumer Academic Publishers, 1990. p. 783-792.

CRUZ, C. F. Foraminíferos e Arcellas (Amebas testáceas) recentes na Baía de Iguape e baixo curso do Rio Paraguaçu: ocorrência e distribuição. Bahia. 2004. 158 f. Dissertação (Mestrado em Geologia) - Universidade Federal da Bahia, Salvador, 2004 $158 \mathrm{p}$.

DAJOZ, R. Ecologia geral. 4. ed. Petrópolis: Vozes, 1983. 475 p.

DAVIES, J. L., A morphogenic approach to world shorelines. Zeitschrift für Geomorphologie, Stuttgart, v. 8, p. 27-42, 1964.

DEBENAY, J. P.; EICHLER, B. B.; BONETTI, C.; COELHO, C. Foraminifers, biomarkers in the mangrove swamps of Bertioga (São Paulo, Brazil). In: REUNIÃO ESPECIAL DA SBPC: ECOSSISTEMAS COSTEIROS - DO CONHECIMENTO À GESTÃO, 3, 1996a, Florianópolis. Resumos... Florianópolis: SBPC, 1996a. p. 448.

DEBENAY, J. P.; EICHLER, B. B.; FERNANDEZ-GONZALEZ, M.; MATHIEU, R.; BONETTI, C.; DULEBA, W. Lês foraminifers paraliques dês Cotes Atlantiques D'Afrique et D'Amerique du Sud de part e d'autre de L'Atlantique, comparasion, discussion. In: JARDINÉ, S.; KLASZ, I.; DEBENAY, J. P. (Ed.). Geologie de L'Afrique et de L'Atlantique Sud. Pau: Elf Aquitaine Edition, 1996b. p. 463-471.

DULEBA, W. Interpretações paleoambientais obtidas a partir das variações na coloração das carapaças de foraminíferos da Enseada do Flamengo. SP. Boletim do Instituto Oceanográfico de São Paulo, São Paulo, v. 42, n. 1/2, p. 63-72, 1994.

DULEBA, W.; DEBENAY, J. P.; EICHLER, B. B. Circulação hidrodinâmica dos estuários da Estação Ecológica JuréiaItatins, inferida a partir das associações de Foraminíferos e Amebas testáceas. In: CUSHMAN FOUNDATION RESEARCH SYMPOSIUM, PDF, 1999, Porto Seguro. Anais... Porto Seguro: VII ABEQUA, 1999. p. 7-9, CC003.

EICHLER-COELHO, P. B.; DULEBA, W.; EICHLER, B. B.; COELHO-JÚNIOR, C. Determinação do impacto ecológico do valo grande (Iguape, SP) a partir das associações de Foraminíferos e Amebas testáceas. Revista Brasileira Biologia, São Carlos, v. 57, n. 3, p. 463-477, 1997

EICHLER, B. B.; CARDOSO, P. B. P. K.; HENKLAIN, F.; EICHLER, P. P. B.; BARCELLOS, R. L. Caracterização ambiental da plataforma interna da região de São Sebastião (SP) durante o Inverno, a partir do estudo de Foraminíferos. In: CONGRESSO DA ASSOCIAÇÃO BRASILEIRA DE ESTUDOS DO
QUATERNÁRIO, VIII, 2001, Imbé. Resumos... Imbé: ABEQUA. 2001. p. 93-95.

ESCOBAR, J. H. J.; MARTINEZ, J. L. Las Arcellas como indicadores ambientales y paleoambientales em águas continentales tropicales: estúdio sistemático del embalse la fé (Antioquia). In: CONGRESSO COLOMBIANO DE GEOLOGIA, VIII, 2002, Medellin. Resumos... Medellin: CCG, 2002. p. 45-47.

GENZ, F.; LESSA, G. C. Caracterização da hidrodinâmica do estuário do rio Paraguaçu em resposta a um hidrograma de cheia gerado pela Barragem de Pedra do Cavalo. In: CONGRESSO BRASILEIRO DE OCEANOGRAFIA; SEMANA NACIONAL DE OCEANOGRAFIA; FEIRA DE CIÊNCIAS E TECNOLOGIA MARINHA, I, XV, I, 2002, Rio de Janeiro. Resumos... Rio de Janeiro: CBO. 2002. p. 33-35.

GREEN, J. Freshwater ecology in the Mato Grosso, Central Brazil, IV: Associations of testace Rhizopoda. Journal of Natural History, London. v. 9, p. 545-549, 1975.

HARDOIM, E. L. Taxonomia e Ecologia de Testacea (Protozoa: Rhizopoda) do Pantanal de Poconé - Rio Bento Gomes e Vazante Birici, Mato Grosso, Brasil. 1997. 343 f. Tese (Doutorado em Ecologia) - Univerdidade Federal de São Carlos, Sorocaba. 1997.

HARDOIM, E. L.; HECKMAN, C. W. The seasonal succession of biotic communities in wetlands of the wet-and-dry climatic zone: IV. The free-living Sarcodines and Ciliates of the Pantanal of Mato Grosso, Brazil. Internacional Review of Hydrobiologie, London, v. 81, p. 367-384, 1996.

KJERFVE, B. Estuarine geomorphology and physical oceanography. In: DAY JR., W.; HALL, C. S. A.; KEMP, W. M.; YÁNES-ARANCIBIA, A. (Ed.). Estuarine ecology. New York: John Wiley \& Sons, 1989. p. 47-78.

KUMAR, A.; DALBY, A. P. Identification key for Holocene Lacustrine Arcellacean (Thecamoebian) Taxa. Ottawa: Press Ottawa-Carleton Geoscience Centre, Department of Earth Sciences, Carleton University, 1998. 34 p.

LANSAC-TÔHA, F. A.; BONECKER, C. C.; VELHO, L. F. M.; IMA, A. F. Composição, distribuição e abundância da comunidade zooplanctônica. In: VAZZOLER, A. E. A. M.; AGOSTINHO, A. A.; HAHN, N. S. (Ed.). Planície de inundação do Alto do Rio Paraná: aspectos físicos, biológicos e socioeconômicos. Maringá: EDUEM, 1997. p. 117-155.

LANSAC-TÔHA, F. A.; VELHO, L. F. M.; BONECKER, C. C. Zooplankton in the Upper Paraná River floodplain: composition, richness, abundance and relationships with the hydrological level and the connectivity. In: AGOSTINHO, A. A. (Ed.). Structure and functioning of the Paraná River and its floodplain. LTER - site 6: (PELD sítio 6). Maringá: EDUEM, 2004. p. 75-84.

LANSAC-TÔHA, F. A.; ZIMMERMANN-CALLEGARI, M. C.; ALVES, G. M.; VELHO, L. F. M.; FULONE, L. J. Species richness and geographic distribution of testate amoebae (Rhizopoda) in Brazilian freshwater environments. Acta Scientiarum Biological. Science, Maringá, v. 29, 185-195, 2007.

LESSA, G. C.; BITTENCOURT, A. C. S. P.; BRICHTA, A.; DOMINGUEZ, J. M. L. The tides and tidal Circulation of Todos os Santos Bay, Northeast Brasil: a general characterization. Anais da Academia Brasileira de Ciências, Rio de Janeiro, v. 73, n. 2, p. 245-261, 2001. 
LOEBLICH, A. R.; TAPPAN, H. Sarcodine, chiefly "Thecamoebians" and "Foraminifera", In: MOORE, R. C. (Ed.). Treatise on invertebrate Paleontology. Protista 2. Pt. C. Kansas: Kansas University Press, 1968. 900 p.

LOEBLICH, A. R.; TAPPAN, H. Foraminifera and Thecamoebians genera and their classification. New York: Van Nostrand Reinold, 1978. 37 p.

MARGALEF, R. Perspectivas de la Teoria Ecológica. Barcelona: Editora Blume, 1958. 110 p.

MASSELINK, G.; FIGUERÊDO, J.; MACHADO, A.; LESSA, G. Foraminífera as na indicator of deposicional environments in a paleo-estuary in NW Austrália. In: CUSHMAN FOUNDATION RESEARCH SYMPOSIUM, VII, 1999, Porto Seguro. Anais... Porto Seguro: ABEQUA, 1999. p. 7-9, CC001.

MEDIOLI, F. S.; SCOTT, D. B. Lacustrine Thecamoebians (Mainly Arcellaceans) as potential tools for paleolimnological interpretations. Paleogeography, Paleoclimatology, Paleoecology, Amsterdam, v. 62, p. 361-386, 1988.

MEDIOLI, F. S.; SCOTT, D. B.; COLLINS, E. S.; McCARTHY, F.M.G. Fossil Thecamoebians present status and prospects for the future. In: HEMLEBEN, C.; KAMINSKI, M. A.; KUNNT, W.; SCOTT, D. B. (Ed.). Paleoecology, Biostratigraphy, Paleoceanography and Taxonomy of agglutinaded Foraminifera, North Atlantic Treaty Organization Advanced Study Institute Serie. Series C. New York: Mathematical and Physical Science, 1990. p. 813-840.

MESTRINHO, S. S. P. Estudo do comportamento geoquímico dos metais pesados nos sedimentos da região estuarina do Rio Paraguaçu - BA. 1998. 158 f. Tese (Doutorado em Oceanografia Geológica) - Universidade de São Paulo, São Paulo. 1998.

MIRANDA, L. B.; CASTRO, B. M.; KJERFVE, B. Princípios de Oceanografia Física de Estuários. São Paulo: Ed. USP, 2002, p. 91122 ,

MURRAY, J. W. Ecology and Paleontology of benthic foraminifera. New York: Longman Scientific \& Technical., 1991. 397 p.

PATTERSON, R.; KUMAR, A. A review of current testate rhizopod (thecamoebian) Research Palaeogeography, Palaeoclimatology, Palaeoecology, Amsterdam, v. 180, p. 225-251, 2002.

PHLEGER, F. B. Sedimentary patterns of microfaunas in northern Gulf of Mexico. Published by The American Association of Petroleum Geologist, Tulsa, p. 267-380, 1960a.

PHLEGER, F. B. Foraminiferal population in Laguna Madre, Texas. Science Reports of the Tohoku University Fourth Series - Biology, v. 4, p. 83-91, 1960b.

ROCHA, A. T. Métodos de estudos dos foraminíferos. Separatas do Boletim do Instituto de Investigação Científica Angola, Lisboa, v. 9, n. 1, p. 27-50, 1972.

SEMENSATTO-JR, D. L. Aplicação dos índices de diversidades em estudos envolvendo associações entre foraminíferos e amebas testáceas recentes: uma breve discussão. In: CONGRESSO SOBRE PLANEJAMENTO E GESTÃO DAS ZONAS COSTEIRAS DOS PAISES DE EXPRESSÃO PROTUGUESA; CONGRESSO DA ASSOCIAÇÃOBRASILEIRO DEESTUDOS DOQUATERNÁRIO; CONGRESSO DO QUATERNÁRIO DOS PAÍSES DE LÍNGUA IBÉRICA, II, IX, II, 2003, Recife. Anais... Recife: ABEQUA, 2003. p. 14-16,

SHANNON, C. E. A mathematical theory of communication. Bell System Technical Journal, Illinois, v. 27, p. 379-423, 1948.
SCOTT, D. B.; SCHAFER, C. T.; MEDIOLI, F. S. Eastern Canadian estuarine foraminifera: a framework for comparision. Journal of Foraminiferal Research, Ithaca, v. 10, n. 3, p. 204-234, 1980.

SCOTT, D. B.; SCHNACK, E. J.; FERRERO, L.; ESPINOSA, M.; BARBOSA, C. F. Recent marsh Foraminifera from the east Coast of South America: comparation to the Northern Hemisphere. In: HEMLEBEN, C.; KAMINSKI, M. A.; KUHNT, W.; SCOTT, D. B. (Ed.). Paleoecology, Biostratigraphy, Paleocenography and Taxonomy of Agglutinated Foraminifera. Dordrecht: Klumer Academic Publishers, 1990. p. 717-738.

SCHÖNBORN, W. Paläolimnologische studien an Testacean aus Bohrkernen des Latnjajaure (Abisko - Gebiet; Schwedisch Lappland). Hydrobiology, Dorcrecht, v. 46, p. 115-139, 1973.

SHARIFI, A. R.; CROUDACE, I. W.; AUSTIN, R. L. Benthic foraminifera as pollution indicators in Southampton Water, Southern England, U.K. Journal of Micropaleontology, Nottingham, v. 10, n. 1, p. 109-113, 1991.

TINOCO, I. M. Introdução ao estudo dos componentes bióticos dos sedimentos marinhos recentes. Recife: Editora Universitária da UFPE, 1989. 219 p.

VALENTIN, J. L. Ecologia numérica: uma introdução à análise multivariada de dados ecológicos. Rio de Janeiro: Interciência, 2000. 117 p.

VELHO, L. F. M.; LANSAC-TÔHA, F. A. Testate Amoebae (Rhizopodea-Sarcodina) from zooplankton of the High Paraná River Floodplain, State Mato Grosso do Sul, Brazil: II. Family Difflugidae. Studies on Neotropical Fauna and Environment, Stuttgart, v. 31, p. 79-192, 1996.

VELHO, L. F. M.; LANSAC-TÔHA, F. A.; BINI, L. M. Spatial and temporal variation in densities of testate amoebae in the plankton of the upper Paraná river. Hydrobiology, Dorcrecht, v. 411, p. $103-$ 113, 1999.

VELHO, L. F. M.; LANSAC-TÔHA, F. A.; BINI, L. M. Influence of environmental heterogeneity on the structure of testate amoebae (Protozoa, Rhizopoda) assemblages in the plankton of the upper Paraná river floodplain, Brazil. Hydrobiology, Dorcrecht, v. 88, p. 154-166, 2003.

VELHO, L. F. M.; LANSAC-TÔHA, F. A.; SERAFIM-JUNIOR, M. Testate Amoebae (Rhizopodea-Sarcodina) from zooplankton of the High Paraná River Floodplain, State of Mato Grosso do Sul, Brazil: I. Families Arcellida and Centropyxidae. Studies on Neotropical Fauna and Environment, Stuttgart, v. 31, p. 35-40, 1996.

WALTER, I. The thecamoebae (Protozoa, Rhizopoda) of small Amazonian forest streams and their possible use as indicators organisms for waterquality. Acta Amazonica, Manaus, v. 12, p. 79105, 1982.

WALTON, W.R. Techniques for recognition of living foraminifera. Contribution of Cushman Fundation for Foraminiferal Research, Washington, v. 3, p. 56-60, 1952.

YANKO, V.; KRONFIELD, J.; FLEXER, A. Response of benthic foraminifera to various pollution sources. Implications for pollution monitoring. Journal of Foraminiferal Research, Ithaca, v. 24, n. 1, p. 1-10, 1994.

ZUCON, M. H.; LOYOLA E SILVA, J. Distribuição espacial de Foraminíferos e Arcellas do Estuário do Rio Piauí, Sergipe. Neritica, Curitiba, v. 7, p. 57-69, 1992. 